\title{
Optimización del proceso de soldadura GMAW de uniones a tope de la aleación AA 6063-T5 basada en la metodología de superficie de respuesta y en la geometría del cordón de soldadura ${ }^{(*)}$
}

\author{
V. Miguel**, E.J. Martínez-Conesa*, F. Segura*, M.C. Manjabacas** y E. Abellán***
}

\begin{abstract}
Resumen La geometría de los cordones de soldadura es caracterizada por el sobreespesor, la anchura y la penetración. Estos valores son indicadores del comportamiento de la unión soldada por lo que pueden considerarse como factores de control del proceso. En el presente trabajo se efectúa la optimización del proceso de soldadura por arco GMAW de la aleación AA 6063-T5 mediante la metodología de la superficie de respuesta (MSR). Las variables consideradas son la tensión del arco, la velocidad de soldeo, la velocidad de alimentación del electrodo y la separación de las superficies a unir. Las funciones de respuesta consideradas son el sobreespesor, anchura, penetración y el ángulo del cordón. Los resultados obtenidos mediante MSR se ajustan muy bien a los valores experimentales. El procedimiento es validado experimentalmente mediante el soldeo, para las condiciones tecnológicas optimizadas obtenidas de forma teórica, existiendo una gran concordancia entre los valores teóricos y los experimentales.
\end{abstract}

Palabras clave Soldadura; Optimización; Aluminio; Metodología de Superficie de Respuesta (MSR); GMAW.

\section{Optimization of GMAW process of AA 6063-T5 aluminum alloy butt joints based on the response surface methodology and on the bead geometry}

\begin{abstract}
The geometry of the weld beads is characterized by the overhead, the width and the penetration. These values are indices of the behavior of the welded joint and therefore, they can be considered as factors that control the process. This work is performed to optimize the GMAW process of the aluminum alloy AA 6063-T5 by means of the response surface methodology (RSM). The variables herein considered are the arc voltage, the welding speed, the wire feed speed and the separation between surfaces in butt joints. The response functions that are herein studied are the overhead, the width, the penetration and the angle of the bead. The obtained results by RSM show high grade of agreement with the experimental values. The procedure is experimentally validated by welding for the theoretically obtained optimized technological conditions and a wide agreement between theoretical and experimental values is found.
\end{abstract}

Keywords

Welding; Optimization; Aluminum; Response Surface Methodology (RSM); GMAW.

\section{INTRODUCCIÓN}

Las propiedades mecánicas de una unión soldada dependen de la geometría del cordón de soldadura, entre otros factores. El estudio de los factores geométricos del cordón de soldadura tiene una consideración importante para el diseño y fabricación de construcciones soldadas. La geometría del cordón de soldadura afecta directamente a la calidad de la sol- dadura en la construcción de estructuras ${ }^{[1]}$. La figura 1 muestra la sección transversal de un cordón de soldadura y su nomenclatura.

La penetración se usa para describir la situación en que el metal depositado y el metal base no se funden de forma integral en la raíz de la soldadura, siempre que estuviera proyectado de este modo. Puede ser ocasionada porque la cara de la raíz de la soldadura no alcance la temperatura de fusión en toda su altura, porque el metal de aportación no llegue a la raíz o

(•) Trabajo recibido el día 9 de diciembre de 2011 y aceptado en su forma final el día 13 de julio de 2012.

* Departamento de Tecnología de Edificación, Universidad Politécnica de Cartagena, Cartagena, España.

** Departamento de Mecánica Aplicada y Proyectos, Universidad de Castilla-La Mancha, Albacete, España.

*** Instituto Politécnico de Cartagena. 


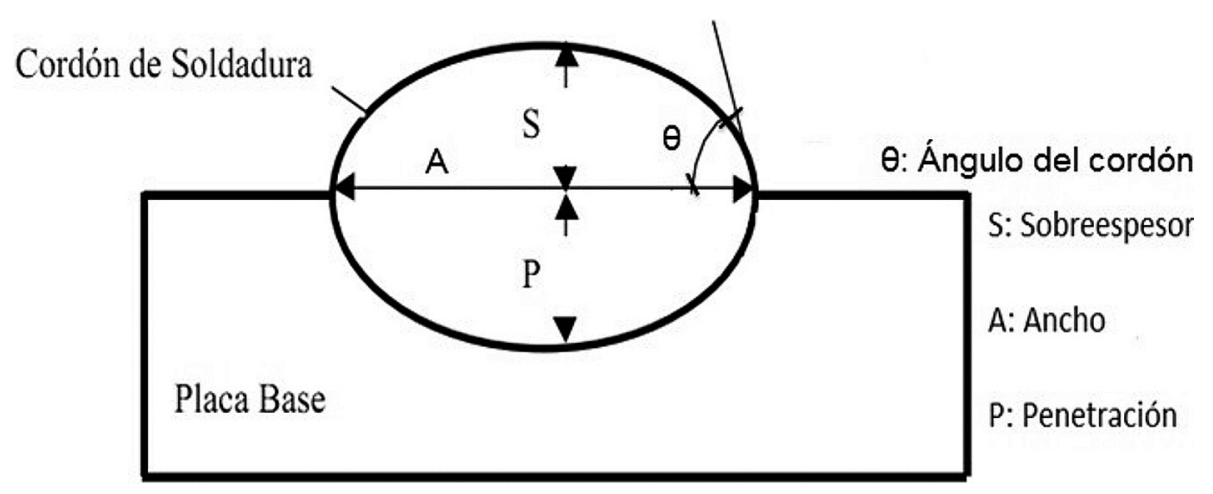

Figura 1. Nomenclatura de la geometría del cordón de soldadura.

Figure 1. Nomenclature of weld bead geometry.

también puede deberse a la falta de disolución de los óxidos e impurezas de la superficie que impiden la unión. No obstante, las dos últimas causas pueden evitarse tomando la precaución de hacer una correcta preparación de bordes y de limpiar las superficies a unir, respectivamente. Las adecuadas condiciones térmicas de la unión hay que abordarlas desde el punto de vista de la investigación de los parámetros que afectan al arco eléctrico con el fin de evitar la penetración incompleta de la unión, que no es deseable, particularmente si la raíz de la soldadura está sometida a solicitaciones.

La geometría del cordón de soldadura juega un papel importante en la determinación de las propiedades mecánicas de una unión soldada. Sus parámetros geométricos como la penetración, el ancho, el sobreespesor y el ángulo del cordón dependen de las variables del proceso de soldadura, tales como la separación entre los bordes a unir, la velocidad de soldadura, la tensión y la velocidad de alimentación del electrodo. Dichos parámetros afectan a la calidad, la productividad y al coste de la unión de estructuras metálicas por soldadura. Por otro lado, la geometría del cordón es de considerable importancia para los ingenieros de diseño ya que afecta a los programas de soldeo, costes de construcción de estructuras y dispositivos mecánicos. Por tanto, el control de los parámetros de soldeo es esencial para conseguir una estructura de calidad.

Por lo tanto, es importante establecer parámetros de soldadura adecuados para producir un cordón de soldadura estable. En general, los criterios de optimización van dirigidos a maximizar la penetración del cordón de soldadura manteniendo los valores de sobreespesor y anchura del cordón lo más bajo posible. Así, para una profundidad de pasada dada, una menor anchura del cordón significa una mayor eficiencia térmica del proceso concentrando más el calor y disminuyendo la zona afectada térmicamente ${ }^{[2]}$. El sobreespesor no es un parámetro determinante desde el punto de vista del comportamiento mecánico de la unión, si bien, un menor sobreespesor significa un mayor rendimiento metalúrgico de la soldadura, esto es, la deposición de metal se realiza con mayor eficiencia. Otro aspecto relacionado con el sobreespesor es que puede actuar como concentrador de tensiones, si resulta excesivo ${ }^{[3]}$, pero los efectos en estructuras con cargas no cíclicas no tienen que verse afectadas significativamente por este motivo. El ángulo del cordón es una consecuencia de la mojabilidad que experimenta el metal líquido sobre el metal base al formar el cordón. En procesos de mojado por gravedad, el ángulo de mojado depende del equilibrio entre las fuerzas de superficie sólido-líquido-gas involucradas en el fenómeno ${ }^{[4]}$, de forma que podría representar, de algún modo, la relación entre el sobreespesor o altura del metal que moja dividido por el área mojada. Ahora bien, en procesos de soldadura por arco, las fuerzas de arrastre de material que tienen lugar en el plasma pueden dar lugar a cordones con una geometría que responda de manera algo diferente. Así, por ejemplo, se podrían obtener cordones planos con cierta anchura, pero con un ángulo de cordón de valor similar a cordones estrechos, cuestión que no está suficientemente estudiada en la bibliografía.

Diversos autores analizan la relación existente entre los parámetros del proceso de soldadura con la geometría de los cordones obtenidos planteando modelos en los que las funciones de respuesta $Y$ se expresan conforme al modelo propuesto por McGlone y Chadwick ${ }^{[5]}$ en función de las variables del proceso $\mathrm{X}_{1}, \mathrm{X}_{2}, \ldots \mathrm{X}_{\mathrm{n}}$, tal y como se indica en la expresión (1) en la que $b_{1} a b_{n}$ son las constantes de ajuste del modelo que dependen del caudal de gas, material de aporte, etc.

$$
\mathrm{Y}=\mathrm{b}_{1} \mathrm{X}_{1}^{\mathrm{b} 1} \mathrm{X}_{2}^{\mathrm{b} 2} \ldots \mathrm{X}_{\mathrm{n}}^{\mathrm{bn}}
$$


Los valores de los coeficientes $b_{1}$ a $b_{n}$ son calculados mediante regresión múltiple. Karandeniz et al. ${ }^{[6]}$, determinan un modelo tipo McGlone y Chadwick, también denominado ajuste curvilíneo, considerando como variables de influencia la tensión e intensidad del arco eléctrico y la velocidad de soldadura. Consideran únicamente el estudio de la penetración por soldadura MAG en aceros de bajo contenido en carbono y se limitan a obtener la variación de dicho parámetro en función de las variables indicadas. Wahab y Painter ${ }^{[7]}$ consideran como variables del proceso la tensión e intensidad del arco, la velocidad de soldadura y el caudal de gas empleado, limitándose a obtener un modelo similar al indicado. Kim et al. ${ }^{[8]}$ también se basan en el modelo curvilíneo, obteniendo como funciones de respuesta la penetración, el sobreespesor y la anchura del cordón. Estos autores establecen que la exactitud de los modelos encontrados varía desde 0 a $25 \%$. En todos los trabajos mencionados hasta el momento, existe como denominador común no solo el modelo empleado sino también que no se establece ninguna metodología de optimización con las variables de respuesta.

Kim et al. ${ }^{[9]}$, correlacionan la penetración del cordón de soldadura con la intensidad, tensión, velocidad de soldadura y ángulo de soldadura. Comparan los resultados obtenidos mediante el modelo curvilíneo con los que se obtienen de una correlación lineal con las variables experimentadas. Los autores demuestran que se comporta mejor el modelo lineal. Concretamente, si se analizan los resultados obtenidos por estos autores, se puede estimar que el error medio cometido en una regresión lineal es del $16 \%$ en tanto que el modelo curvilíneo que representa la expresión (1) conduce a un error medio del $23 \%$. Ambos métodos presentan algún valor experimental que se aleja en más del $50 \%$ del correspondiente modelo teórico. El ángulo de soldeo experimentado varía de $10^{\circ}$ a $20^{\circ}$, que es el rango de aplicación típico en operaciones de soldadura GMAW robotizadas.

Existen autores que emplean correlaciones de la geometría del cordón con el valor de energía aportada en el proceso. Murray y Scotti ${ }^{[10]}$ demuestran que la profundidad del cordón de soldadura en procesos GMAW de acero inoxidable depende del modo de transferencia del electrodo al metal, estableciendo una correlación en función de los parámetros transferencia de masa y transferencia de calor adimensionales. La correlación encontrada permite determinar la profundidad del cordón para diferentes modos de transferencia de material. Dorta et al. [11] obtienen valores de la geometría del cordón para soldadura de aceros inoxidables dúplex en función de la tensión, la velocidad de soldeo y de la intensidad de corriente. También evalúan la correlación exis- tente entre la geometría y la energía aportada. De este modo cuantifican la tensión, la intensidad de corriente y la velocidad de soldeo en una sola variable, obteniendo índices de correlación significativos. Ello permite establecer que los efectos de una de las variables no son contrarrestados por las otras. Las funciones de correlación obtenidas son de tipo curvilíneo o polinómico.

Payares et al. ${ }^{[12]}$ determinan el área del cordón de soldadura en función de la tensión, la intensidad y la velocidad de soldeo en soldaduras a tope de la aleación de aluminio 6063-T5. Obtienen funciones de tipo curvilíneo para el área transversal estimada del cordón de soldadura con los parámetros tecnológicos del proceso.

Existen pocas investigaciones publicadas en relación a la geometría del cordón de soldadura en aleaciones de aluminio. A pesar del interés que suscita en la construcción de estructuras ${ }^{[13]}$, no existen investigaciones dirigidas a la optimización de los diversos parámetros tecnológicos que intervienen en el proceso conjuntamente y se suelen establecer simplificaciones en la ejecución de cordones de soldadura que no tienen en cuenta, por ejemplo, la separación entre las superficies a unir. En el presente trabajo, se lleva a cabo la modelización del proceso de soldadura de la aleación de aluminio 6063-T5 mediante la metodología de superficie de respuesta para soldaduras a tope con diferentes grados de separación entre superficies. A partir del modelo obtenido se optimiza el proceso y se realiza la validación experimental de las condiciones de optimización.

\section{DISEÑO DE EXPERIMENTOS MEDIANTE LA METODOLOGÍA DE SUPERFICIE DE RESPUESTA (MSR)}

La metodología de superficie de respuesta (MSR) es un conjunto de técnicas matemáticas y estadísticas que son útiles para la modelización y análisis en aplicaciones en las que una respuesta de interés es influenciada por distintas variables y cuyo objetivo es optimizar esta respuesta.

El modelo de respuesta cuadrático o modelo de segundo orden, es el indicado en la expresión (2):

$y=\beta_{0}+\sum_{i=1}^{k} \beta_{i} X_{i} \sum_{i=1}^{k} \beta_{i i} X_{i}^{2}+\sum_{j=2} \sum_{i=1}^{k} \beta_{i j} X_{i} X_{j}+\varepsilon$

Aplicado a la soldadura por arco eléctrico, se puede estudiar cómo los parámetros del procedimiento de soldeo afectan a la penetración, sobrees- 
pesor, anchura del cordón y ángulo del cordón de una unión soldada y permite encontrar los valores que optimicen esta respuesta. Esto es, se trata de obtener los valores óptimos para las variables independientes que maximizan, minimizan o cumplen ciertas restricciones en la variable respuesta. Concretamente, se precisa determinar los niveles de cuatro variables, $x_{1}$ separación entre piezas, $x_{2}$ velocidad de soldadura, $x_{3}$ tensión de soldeo y $x_{4}$ velocidad de alimentación del electrodo, que maximizan la penetración y minimizan el ancho, el sobreespesor y el ángulo del cordón en el metal base. Tanto la penetración, $y_{1}$, como el ancho, $y_{2}$, el sobreespesor, $y_{3}$, y el ángulo del cordón, $y_{4}$, se pueden expresar como funciones de las variables indicadas y del error observado, tal y como se establece de forma genérica para la función $\mathrm{y}_{\mathrm{i}}$ en la expresión (3):

$$
y_{i}=f\left(x_{1}, x_{2}, x_{3}, x_{4}\right)+\varepsilon_{i}
$$

En el presente trabajo se ha adoptado un modelo cuadrático, estableciendo la relación entre los factores y niveles $(-1,0,1)$ que se consideran en la tabla I.

Con la combinación de los valores de las variables indicados en la tabla II se ha realizado la matriz de experimentos, teniendo en cuenta los valores codificados de las variables conforme a los niveles $(-1,0,1)$.

El intervalo de valores seleccionados para las variables corresponde a valores reales utilizados en los procedimientos de soldeo de estructuras metálicas. En la tabla II se indican los experimentos seleccionados para la obtención de la matriz de experimentos y se especifican los valores correspondientes a cada variable en los diferentes experimentos considerados. Se realizan los experimentos correspondientes a los valores de las variables indicados en la tabla II y se obtienen los parámetros geométricos indicados en la tabla III.

Tabla I. Niveles de las variables del proceso de soldadura por arco eléctrico según MSR

Table I. Levels of process variables in arc welding as RSM

\begin{tabular}{lccc}
\hline Parámetros de soldeo & $\mathbf{- 1}$ & $\mathbf{0}$ & $\mathbf{1}$ \\
\hline Separación entre piezas $\left(\mathrm{x}_{1}\right)(\mathrm{mm})$ & 0 & 1 & 2 \\
Velocidad de soldadura $\left(\mathrm{x}_{2}\right)(\mathrm{mm} / \mathrm{s})$ & 8,2 & 13 & 17,8 \\
Tensión $\left(\mathrm{x}_{3}\right)(\mathrm{V})$ & 18 & 21 & 24 \\
Velocidad del electrodo $\left(\mathrm{x}_{4}\right)(\mathrm{mm} / \mathrm{s})$ & 9 & 10,5 & 12 \\
\hline
\end{tabular}

Tabla II. Valores de las variables considerados para la elaboración de la matriz de experimentos

Table II. Values of the variables considered in the preparation of the matrix of experiments

\begin{tabular}{cccccccccc}
\hline Cordón & $\begin{array}{c}\mathbf{x}_{\mathbf{1}} \\
(\mathbf{m m})\end{array}$ & $\begin{array}{c}\mathbf{x}_{\mathbf{2}} \\
(\mathbf{m m} / \mathbf{s})\end{array}$ & $\begin{array}{c}\mathbf{x}_{\mathbf{3}} \\
(\mathbf{V})\end{array}$ & $\begin{array}{c}\mathbf{x}_{\mathbf{4}} \\
(\mathbf{m} / \mathbf{m i n})\end{array}$ & Cordón & $\begin{array}{c}\mathbf{x}_{\mathbf{1}} \\
(\mathbf{m m})\end{array}$ & $\begin{array}{c}\mathbf{x}_{\mathbf{2}} \\
(\mathbf{m m} / \mathbf{s})\end{array}$ & $\begin{array}{c}\mathbf{x}_{\mathbf{3}} \\
(\mathbf{V})\end{array}$ & $\begin{array}{c}\mathbf{x}_{\mathbf{4}} \\
(\mathbf{m} / \mathbf{m i n})\end{array}$ \\
\hline 1 & 1 & 13 & 18 & 12 & 13 & 0 & 13 & 21 & 9 \\
2 & 1 & 13 & 24 & 12 & 14 & 1 & 17,8 & 18 & 10,5 \\
3 & 2 & 13 & 24 & 10,5 & 15 & 0 & 17,8 & 21 & 10,5 \\
4 & 2 & 13 & 21 & 12 & 16 & 1 & 8,2 & 21 & 12 \\
5 & 2 & 17,8 & 21 & 10,5 & 17 & 2 & 8,2 & 21 & 10,5 \\
6 & 1 & 17,8 & 24 & 10,5 & 18 & 1 & 17,8 & 21 & 12 \\
7 & 0 & 13 & 18 & 10,5 & 19 & 1 & 13 & 24 & 9 \\
8 & 0 & 8,2 & 21 & 10,5 & 20 & 1 & 8,2 & 18 & 10,5 \\
9 & 1 & 13 & 18 & 9 & 21 & 1 & 8,2 & 21 & 9 \\
10 & 2 & 13 & 18 & 10,5 & 22 & 1 & 8,2 & 24 & 10,5 \\
11 & 0 & 13 & 21 & 12 & 23 & 1 & 17,8 & 21 & 9 \\
12 & 0 & 13 & 24 & 10,5 & 24 & 2 & 13 & 21 & 9 \\
\hline
\end{tabular}


Tabla III. Valores de los parámetros geométricos obtenidos tras el procedimiento experimental Table III. Values of the geometric parameters obtained after the experimental procedure

\begin{tabular}{ccrrrrrrrr}
\hline Cordón & $\begin{array}{c}\mathbf{Y}_{\mathbf{1}} \\
(\mathbf{m m})\end{array}$ & $\begin{array}{c}\mathbf{Y}_{\mathbf{2}} \\
(\mathbf{m m})\end{array}$ & $\begin{array}{c}\mathbf{Y}_{\mathbf{3}} \\
(\mathbf{m m})\end{array}$ & $\begin{array}{c}\mathbf{Y}_{\mathbf{4}} \\
\mathbf{(} \mathbf{)}\end{array}$ & Cordón & $\begin{array}{c}\mathbf{Y}_{\mathbf{1}} \\
(\mathbf{m m})\end{array}$ & $\begin{array}{c}\mathbf{Y}_{\mathbf{2}} \\
(\mathbf{m m})\end{array}$ & $\begin{array}{c}\mathbf{Y}_{\mathbf{3}} \\
(\mathbf{m m})\end{array}$ & $\begin{array}{c}\mathbf{Y}_{\mathbf{4}} \\
(\mathbf{(})\end{array}$ \\
\hline 1 & 5,067 & 7,957 & 2,995 & 55 & 13 & 2,039 & 8,958 & 2,433 & 44 \\
2 & 3,850 & 10,984 & 2,540 & 35 & 14 & 3,748 & 6,733 & 2,037 & 46 \\
3 & 4,313 & 7,584 & 0,954 & 23 & 15 & 2,390 & 8,327 & 2,311 & 51 \\
4 & 4,353 & 9,122 & 2,161 & 27 & 16 & 4,682 & 12,110 & 3,772 & 47 \\
5 & 3,972 & 6,038 & 0,663 & 20 & 17 & 4,954 & 10,920 & 2,265 & 55 \\
6 & 3,067 & 7,407 & 1,771 & 50 & 18 & 4,284 & 7,790 & 1,876 & 27 \\
7 & 2,281 & 7,718 & 2,740 & 61 & 19 & 2,358 & 9,885 & 1,675 & 28 \\
8 & 2,612 & 10,996 & 3,552 & 57 & 20 & 3,725 & 8,964 & 2,932 & 63 \\
9 & 1,572 & 6,901 & 2,073 & 41 & 21 & 3,401 & 8,900 & 2,642 & 55 \\
10 & 5,394 & 7,729 & 1,512 & 48 & 22 & 3,841 & 12,804 & 3,118 & 37 \\
11 & 3,609 & 8,502 & 2,687 & 54 & 23 & 2,898 & 7,403 & 1,131 & 30 \\
12 & 2,113 & 9,363 & 2,380 & 50 & 24 & 3,752 & 7,841 & 1,099 & 24 \\
\hline
\end{tabular}

\section{PROCEDIMIENTO EXPERIMENTAL}

El material utilizado en los ensayos es la aleación de aluminio AA 6063-T5, en condiciones de soldeo a temperatura ambiente. Las dimensiones de los cupones de soldadura fueron de $100 \times 40 \times 8 \mathrm{~mm}^{3}$. El punto de fusión para el aluminio ensayado se estima en el intervalo de $615^{\circ} \mathrm{C}-655^{\circ} \mathrm{C}$ y la composición química se indica en la tabla IV.

En la tabla $\mathrm{V}$ se recopilan las propiedades físicas típicas (a temperatura ambiente de $20^{\circ} \mathrm{C}$ ). El procedimiento de soldadura empleado fue GMAW.

El gas de protección seleccionado fue argón comercial al 99 \% típico de estas aplicaciones y el caudal empleado 14 l/min. La distancia entre la antorcha y

Tabla IV. Composición química (\%) de la aleación de aluminio AA 6063-T5

Table IV. Chemical composition (\%) of the aluminum alloy AA 6063-T5

\begin{tabular}{ccccccccc}
\hline Mn & Si & Cr & $\mathrm{Cu}$ & $\mathrm{Zn}$ & $\mathrm{Pb}$ & $\mathrm{Fe}$ & $\mathrm{Ti}$ & $\mathbf{M g}$ \\
\hline 0,020 & 0,430 & 0,010 & 0,010 & 0,010 & 0,010 & 0,200 & 0,020 & 0,480 \\
\hline
\end{tabular}

Tabla V. Propiedades físicas típicas (a temperatura ambiente de $20^{\circ} \mathrm{C}$ ) de la aleación de aluminio AA 6063-T5

Table V. Typical physical properties (at room temperature of $20^{\circ} \mathrm{C}$ ) of the aluminum alloy AA 6063-T5

\begin{tabular}{ccccccc}
\hline $\begin{array}{c}\text { Módulo } \\
\text { elástico } \\
\text { (MPa) }\end{array}$ & $\begin{array}{c}\text { Límite } \\
\text { elástico } \\
\text { (MPa) }\end{array}$ & $\begin{array}{c}\text { Resistencia } \\
\text { a la rotura } \\
(\mathbf{M P a})\end{array}$ & $\begin{array}{c}\text { Alargamiento } \\
\text { de rotura } \\
\mathbf{( \% )}\end{array}$ & $\begin{array}{c}\text { Peso } \\
\text { específico } \\
\left(\mathbf{g} / \mathbf{c m}^{3}\right)\end{array}$ & $\begin{array}{c}\text { Intervalo } \\
\text { de fusión } \\
\left.\mathbf{(}{ }^{\circ} \mathbf{C}\right)\end{array}$ & $\begin{array}{c}\text { Coef. } \\
\text { dilatación } \\
\text { lineal } \\
(\mathbf{1 / 1 0 6} \mathbf{K})\end{array}$ \\
\hline 69.500 & 145 & 185 & 22 & 2,70 & $615-655$ & 23,5 \\
\hline
\end{tabular}


la superficie de soldeo se mantuvo en $3 \mathrm{~mm}$ para garantizar la protección del gas. El electrodo empleado corresponde a la denominación S Al 5356 $\left(\mathrm{AlMg}_{5} \mathrm{Cr}(\mathrm{A})\right)$ conforme a la norma EN ISO 18273 , de 1,2 $\mathrm{mm}$ de diámetro. Dicho electrodo es típico de aplicaciones generales.

Para la sujeción correcta de las probetas se realizó un soporte de aluminio que se alojó en un robot pórtico y cuyo objetivo era la colocación de los cupones a soldar y su fijación mediante pernos. En la figura 2 se observa un detalle del dispositivo descrito.

Antes del proceso de soldeo, las zonas a unir son tratadas superficialmente con un cepillo de púas de aluminio para eliminar cualquier tipo de oxidación superficial que dificulte la estabilidad del arco eléctrico durante la soldadura. Para controlar la separación entre las piezas se utilizan chapas de 1 y 2 mm de espesor. Los parámetros de tensión y velocidad de alimentación del electrodo son regulados mediante los controles existentes en la máquina de soldadura
(TransPuls Synergic). La velocidad de soldadura se establece mediante el desplazamiento del robot pórtico, que es programado por control numérico. Las probetas soldadas se cortan por la mitad mediante la sierra de cinta con refrigeración para evitar calentamientos en el corte, siendo convenientemente lijadas con papeles de granulometría 1000 que conducen a un acabado suficiente para ejecutar la medición de los parámetros geométricos mediante el empleo de un proyector de perfiles.

En la figura 3 se muestra un detalle de algunos de los cordones realizados.

De manera aproximada se puede considerar el área del cordón de soldadura y la parte del metal base que participa del mismo, es decir, se puede estimar la dilución existente, aunque no es objeto del presente trabajo.

Los resultados experimentales obtenidos para los valores de penetración, anchura, sobreespesor y ángulo de cordón para las diferentes condiciones de

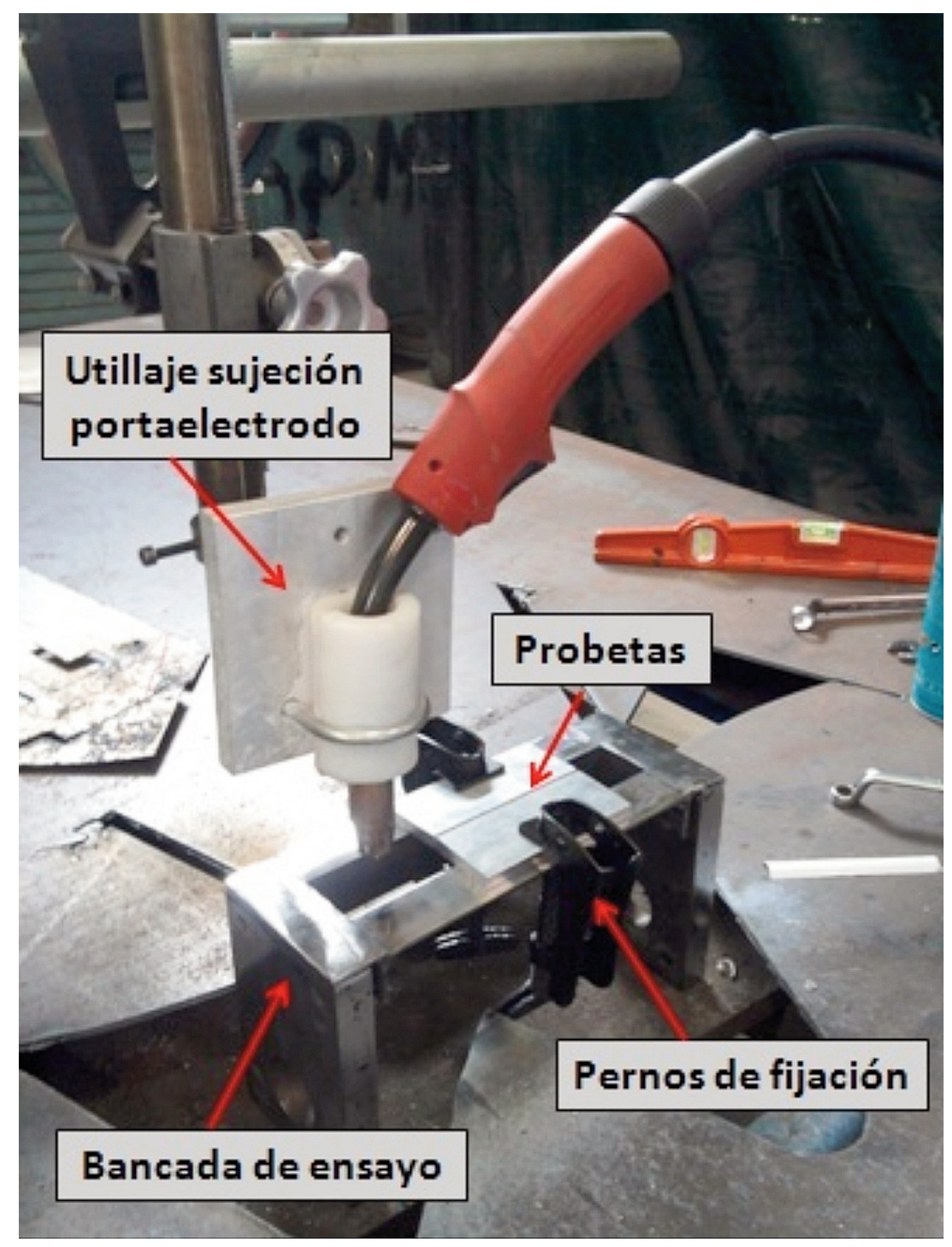

Figura 2. Equipo experimental.

Figure 2. Experimental equipment. 

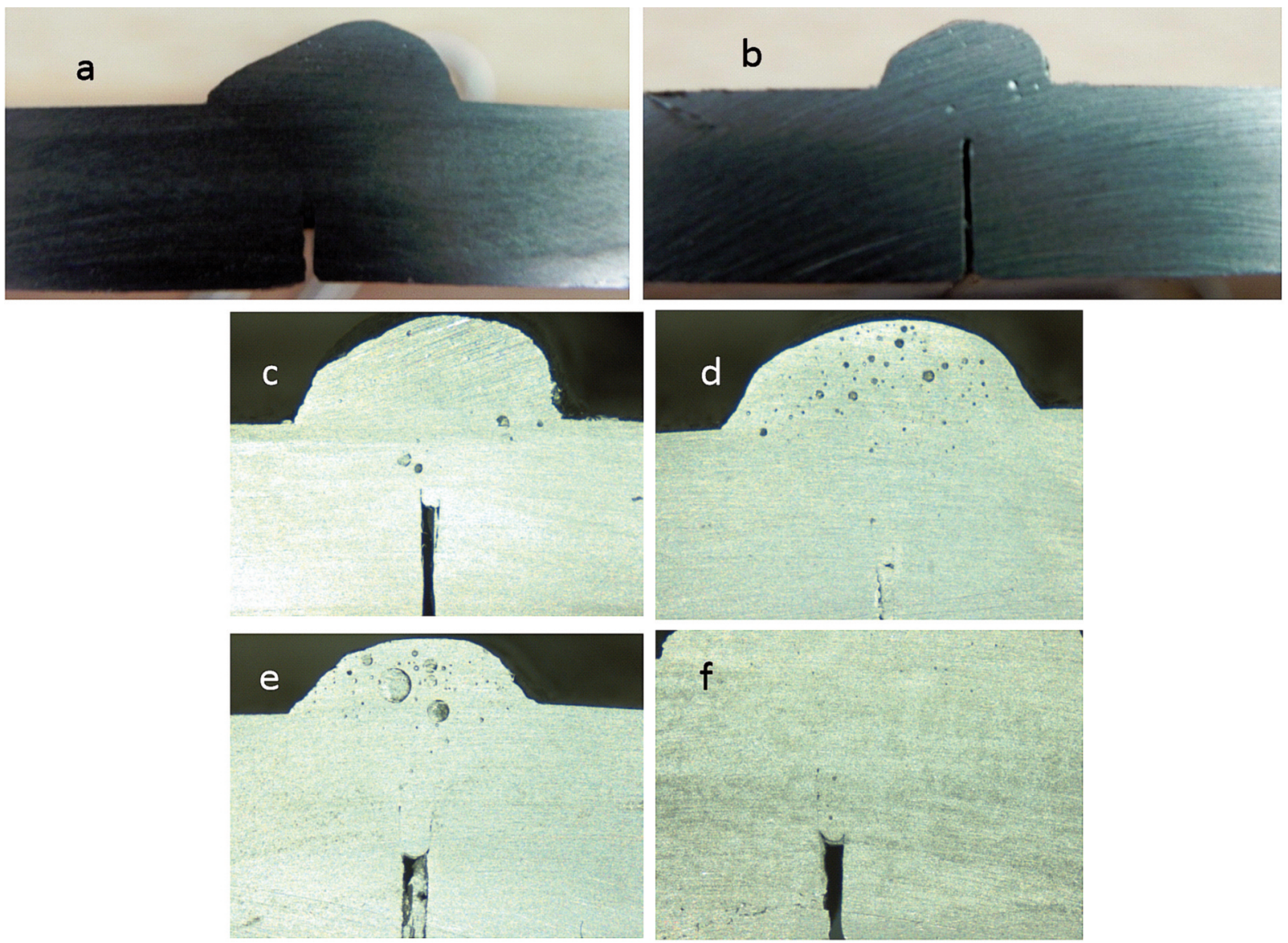

Figura 3. Vista general de secciones transversales de cordones de soldadura. a) Ensayo 16; b) ensayo 7; c) ensayo 7(detalle 4x); d) ensayo 12 (detalle 4x); e) ensayo 14 (detalle 4x); f) ensayo 16 (detalle $4 x$ ).

Figure 3. Transversal views of different weld beads. a) Test 16; b) test 7 ; c) test 7 (detail 4x); d) test 12 (detail 4x); e) test 14 (detail 4x); f) test 16 (detail 4x).

soldeo se establecen en la figura 4 , junto con los valores que se obtienen mediante la modelización MSR conforme al epígrafe siguiente.

\section{RESULTADOS MSR}

El MSR se plantea en forma matricial conforme se indica en la expresión (4). La resolución de dicha ecuación va dirigida a la obtención de la matriz de coeficientes, [B].

$$
\left[\mathrm{Y}_{1}\right]=[\mathrm{X}][\mathrm{B}]
$$

La ecuación matricial se ha resuelto mediante el empleo de la aplicación informática "Sagata", obteniendo como resultado de la modelización matemática entre las variables de entrada y la respuesta del método las expresiones correspondientes a las variables de salida. En la figura 4 se establecen los valores obtenidos para los elementos de la geometría del cordón de soldadura.

Para la penetración, se obtiene la ecuación (5). En dicha ecuación $S$ representa la separación entre piezas, $V_{s}$ la velocidad de soldadura, $T$ la tensión eléctrica de soldeo y $V_{e}$ la velocidad de alimentación del electrodo.

$Y_{1}=-34,6593+4,8033 S+0,0012 V_{S}+$

$+1,6747 T+2,9969 V_{\varepsilon}-0,0104 S^{2}+$

$+0,0093 V_{s}^{2}-0,0074 T^{2}-0,0396 S V_{s}-$

$-0,0761 S T-0,0138 V_{s} T-0,1615 S V_{\varepsilon}+$

$+0,0036 V_{s} V_{\varepsilon}-0,113 T V_{\varepsilon}$

En la figura 5 se representan las gráficas de la superficie de respuesta para la penetración en función de las variables tecnológicas del proceso de soldeo.

Se puede observar que las variables con mayor influencia sobre la penetración son la separación de las superficies de soldadura y la velocidad de alimen- 


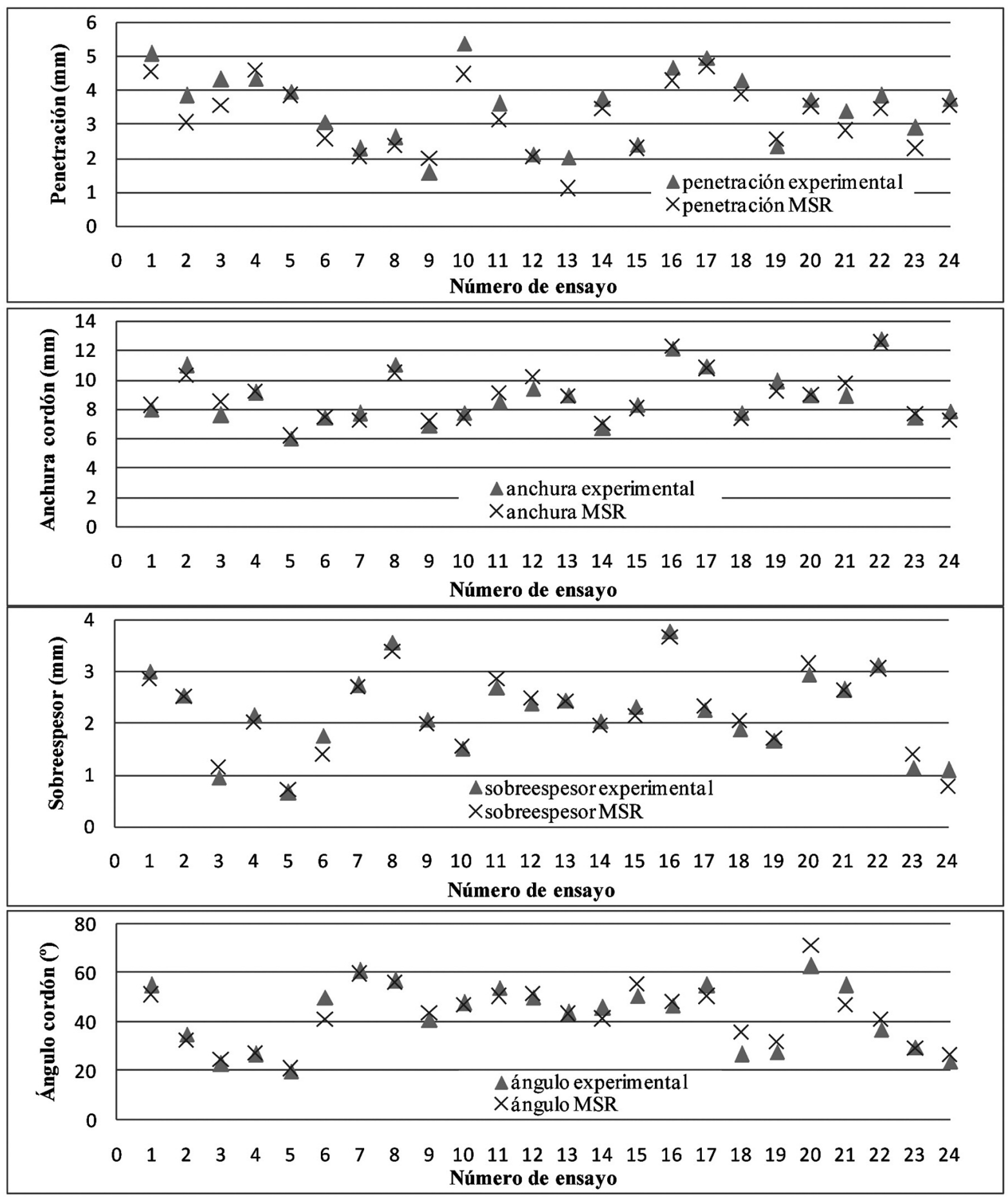

Figura 4. Valores de los elementos de la geometría del cordón de soldadura obtenidos experimentalmente y mediante la modelización MSR para las condiciones de cada uno de los experimentos efectuados.

Figure 4. Values for the different geometric elements for each weld obtained experimentally and RSM modeling.

tación del electrodo, siendo la variación obtenida lineal en ambos casos. En relación a la separación, se obtiene una penetración mayor conforme la separación aumenta, tal y como era esperable.
En la figura 6 se observa que conforme aumenta la velocidad de soldadura la penetración disminuye rápidamente, pero se mantiene prácticamente invariable para los mayores valores de velocidad de soldeo. 

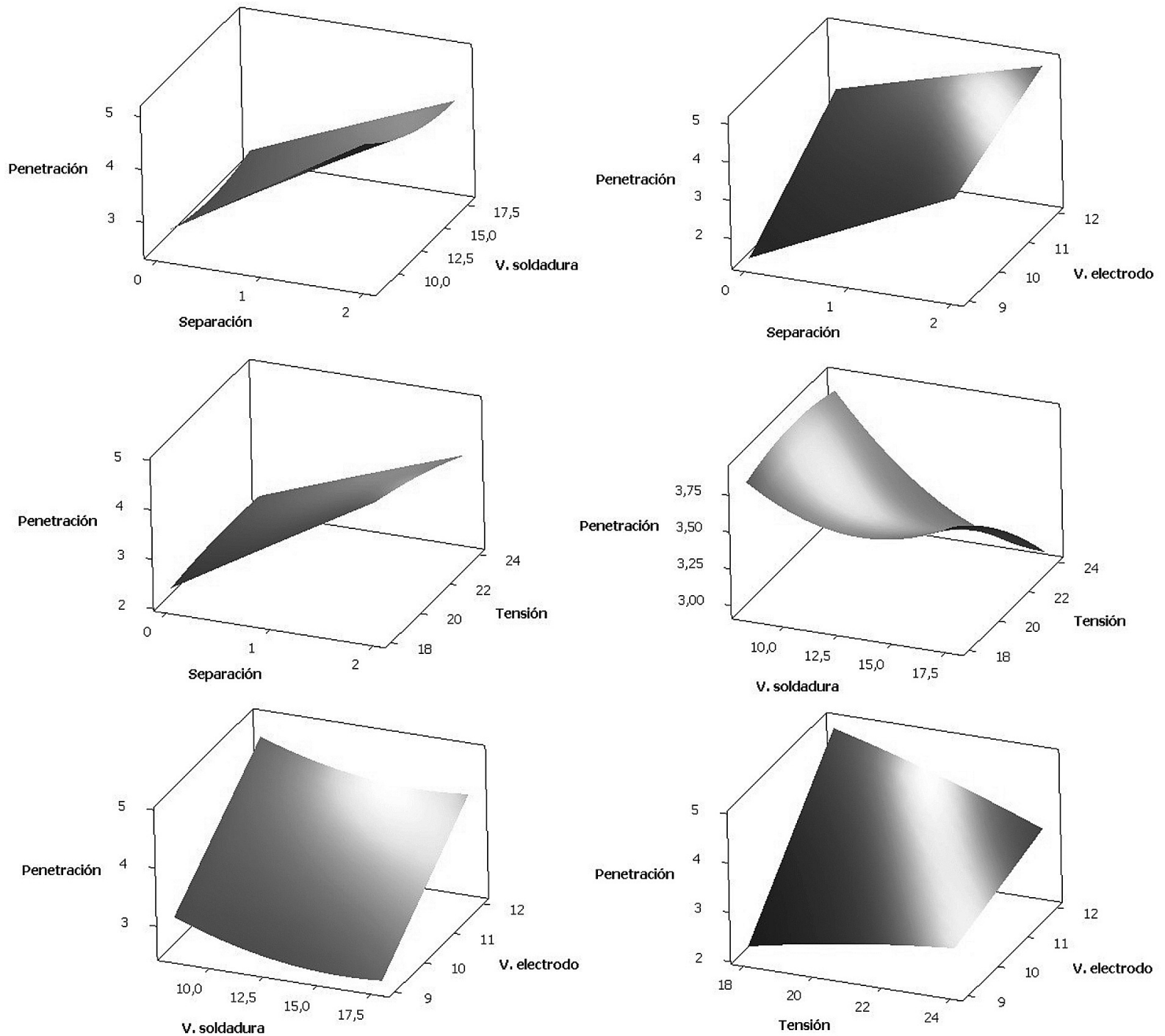

Figura 5. Gráficas de superficie de respuesta de la penetración en función de los factores tecnológicos del proceso GMAW.

\section{Figure 5. Response surface graphs of the penetration as a function of the technological parameters of the GMAW process.}

Con la tensión del arco, la penetración tiende a disminuir. Ambas tendencias son esperables de acuerdo con los principios de la soldadura por arco eléctrico. Efectivamente, una mayor velocidad de soldeo disminuye la energía neta aportada y con ello la penetración. Un aumento de la tensión implica un enfriamiento del arco eléctrico, teniendo en cuenta las curvas de características eléctricas de estos equipos, lo que conduce también a disminuir la penetración. Además, la forma en que disminuye la intensidad de corriente con un aumento de la tensión es también lineal en este tipo de equipos ${ }^{[14]}$, lo que resulta coherente con los resultados obtenidos, habida cuenta de que la relación de la intensidad, esto es, la velocidad de alimentación del electrodo, es también lineal con la penetración.
En definitiva, se observa que los factores que más afectan al aumento de la penetración son la separación y la velocidad de alimentación del electrodo.

La expresión para la anchura del cordón queda modelizada como se establece en la ecuación (6).

$Y_{2}=38,9380+2,0191 S+1,6466 V_{s}+$

$+2,5283 T+1,30 V_{e}-0,4069 S^{2}+$

$+0,0111 V_{s}^{2}-0,0323 T^{2}-0,1153 S V_{s}-$

$-0,1492 S T-0,0550 V_{s} T+0,2895 S V_{e}-$

$-0,0980 V_{s} V_{e}+0,0024 T V_{\varepsilon}$

En la figura 7 se observa cómo el factor más determinante en el ancho del cordón es la velocidad de 


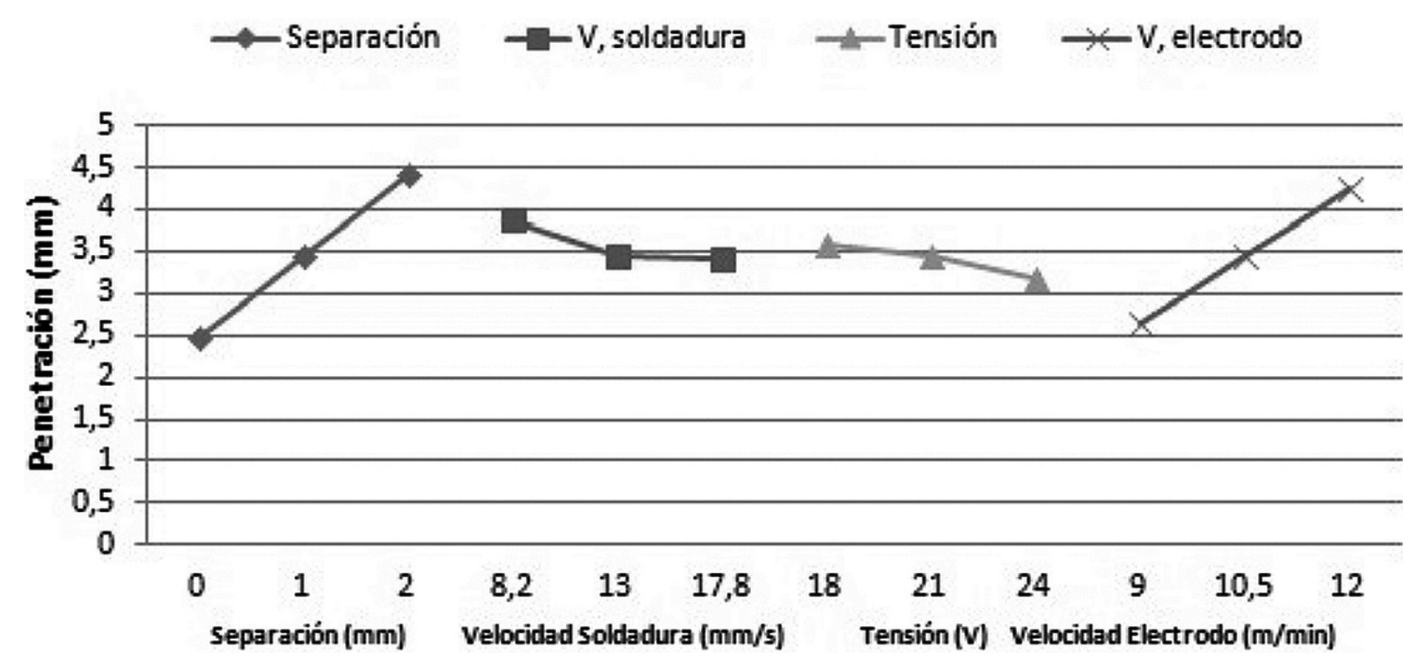

Figura 6. Penetración en función de los factores tecnológicos del proceso GMAW.

Figure 6. Penetration as a function of technological factors of the GMAW process.
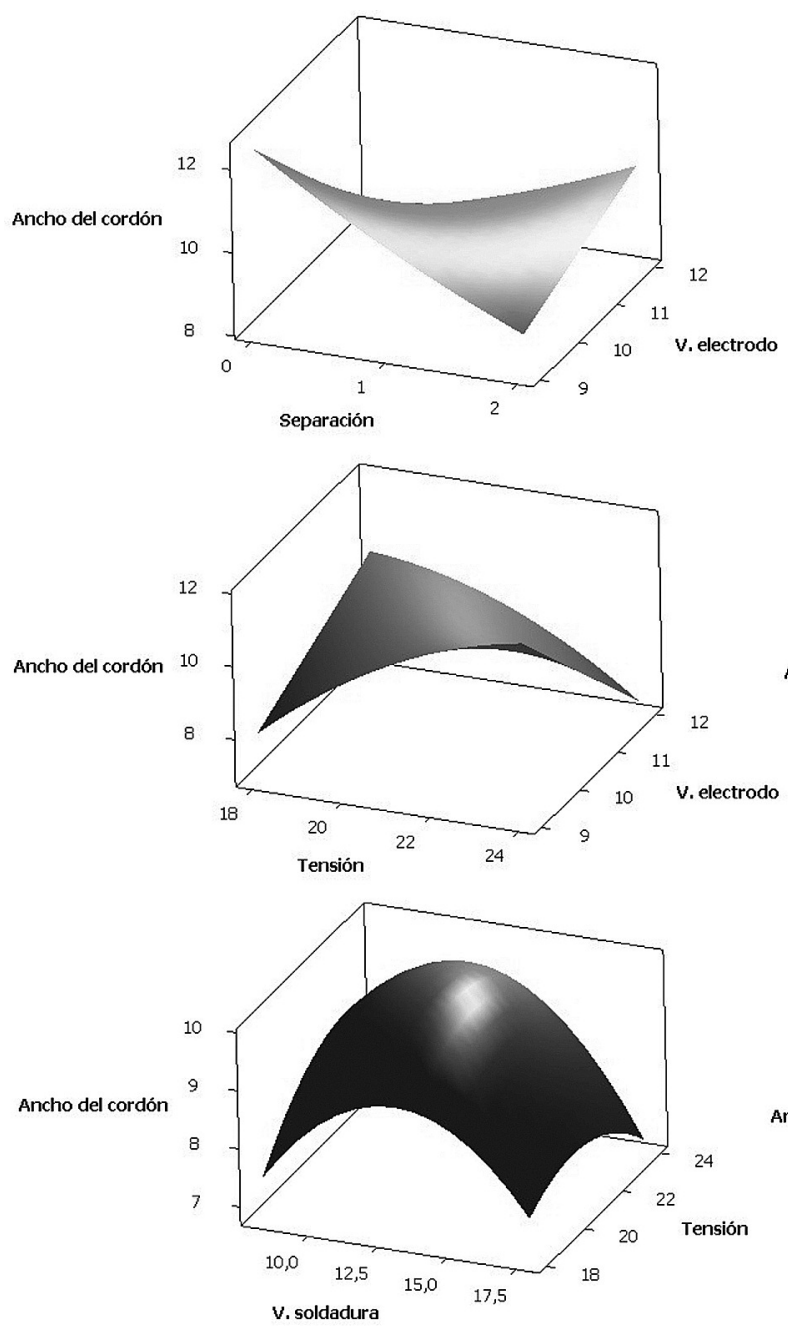
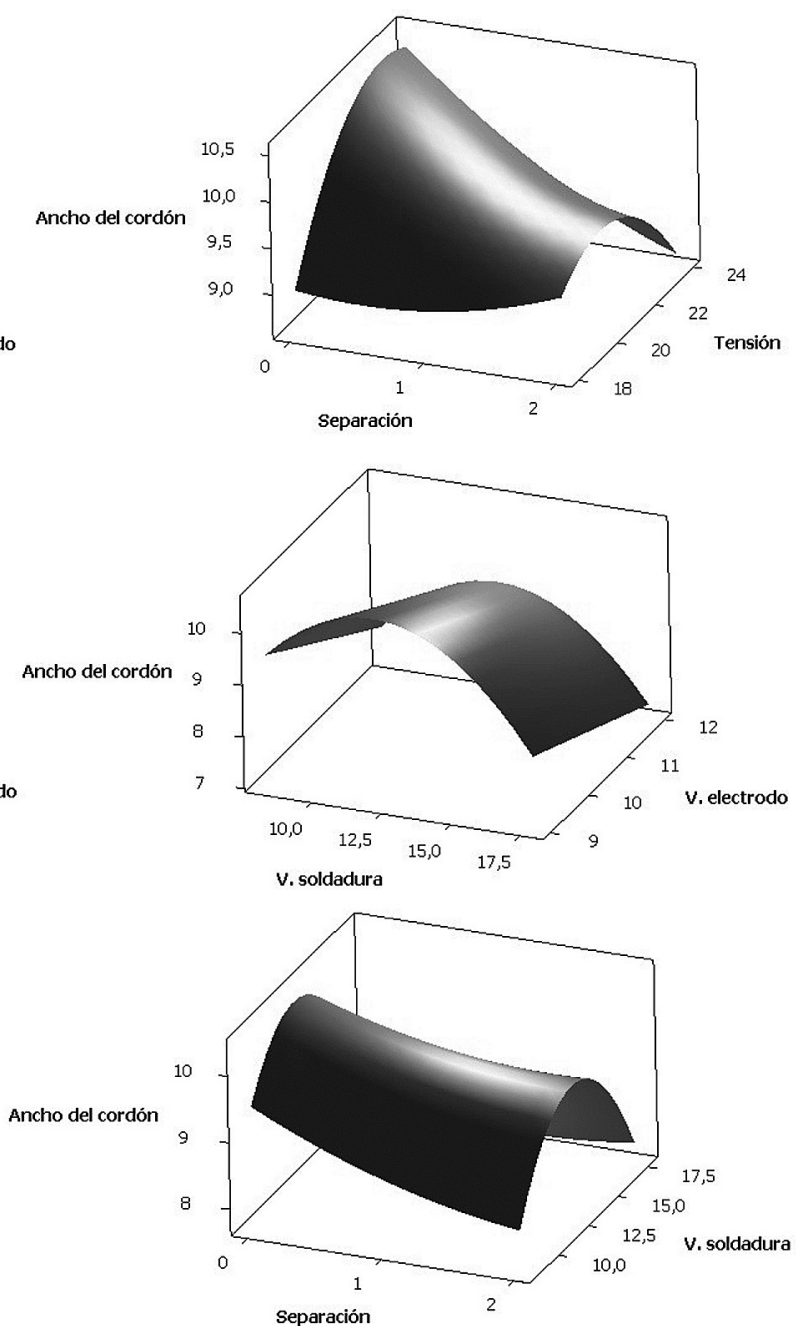

Figura 7. Gráficas de superficie de respuesta del ancho del cordón en función de los factores tecnológicos del proceso GMAW.

Figure 7. Response surface graphs of bead width as a function of technological factors of the GMAW process. 
soldadura, de manera que se obtiene un ancho mínimo conforme la velocidad de soldadura aumenta.

En la figura 8 se observa que el ancho del cordón disminuye conforme aumenta la separación.

En el caso de la velocidad de soldadura, pasa lo mismo: conforme aumenta la velocidad el ancho disminuye considerablemente; se ve que la pendiente es significativa, por ello, este factor es el más importante (junto con la tensión), puesto que controlándolo se obtiene un cordón relativamente fino. En el caso de la tensión, se observa que con una tensión alta el ancho aumenta de forma considerable y es, como se ha dicho anteriormente, un factor determinante para obtener un cordón óptimo. Observando la velocidad de alimentación del electrodo indica que, al igual que en la penetración, al usar una mayor velocidad, se obtiene un aumento de forma progresiva del ancho del cordón. Desde un punto de vista térmico se observa una contradicción de la tendencia de comportamiento de la anchura del cordón con la tensión y la intensidad. Es sabido que una alta intensidad favorece el aplanado del cordón, con mayor anchura y penetración que para los valores de intensidad bajos. No obstante, tal y como se ha comentado con anterioridad los valores elevados de tensión conducen a intensidades de arco menores y, en consecuencia a arcos algo más fríos. Por tanto, el efecto de la tensión debe interpretarse desde el punto de vista del fundamento físico del arco como chorro de plasma con arrastre del material desde el electrodo hasta la zona de unión. Una mayor tensión favorece fuerzas de arrastre mayores hacia la superficie de la zona de unión, lo que se traduce en cordones más anchos y planos, esto es, con menor sobreespesor, tal y como se confirma en las figuras 9 y 10 , que corresponden a la definición de esta última variable.

La ecuación para el sobreespesor queda modelizada tal y como se establece en la expresión (7).

$Y_{3}=-2,3746-0,8621 S+0,0374 V_{s}+$

$+0,3086 T+0,3822 V_{e}-0,2909 S^{2}+$

$+0,0053 V_{s}^{2}-0,0050 T^{2}-0,0188 S V_{s}-$

$-0,0165 S T-0,0078 V_{s} T+0,1347 S V_{\varepsilon}-$

$-0,0134 V_{s} V_{\varepsilon}-0,0032 T V_{\varepsilon}$

Las figuras 9 y 10 representan las superficies de respuesta y de acciones descompuestas correspondientes al sobreespesor.

En la figura 9 se observa que los factores más determinantes en el sobreespesor son la velocidad de soldadura y la separación; se obtiene un sobreespesor mínimo conforme ambos factores aumentan.

En definitiva, se observa que el sobreespesor disminuye conforme aumenta la separación (Fig. 10). El sobreespesor mínimo se obtiene empleando una velocidad de soldadura alta. La tensión tiene tendencia a disminuir el sobreespesor. Por último, una velocidad de electrodo elevada, o lo que es lo mismo, una alta intensidad, conduce a la obtención de mayores valores para el sobreespesor. Todas las tendencias observadas son justificables desde el punto de vista de los principios de comportamiento de los procesos de soldadura por arco. La influencia de la tensión de soldadura, tal y como se ha establecido con anterioridad, debe justificarse a partir de la fuerza de arrastre del material, mayor para tensiones elevadas, que establece un equilibrio

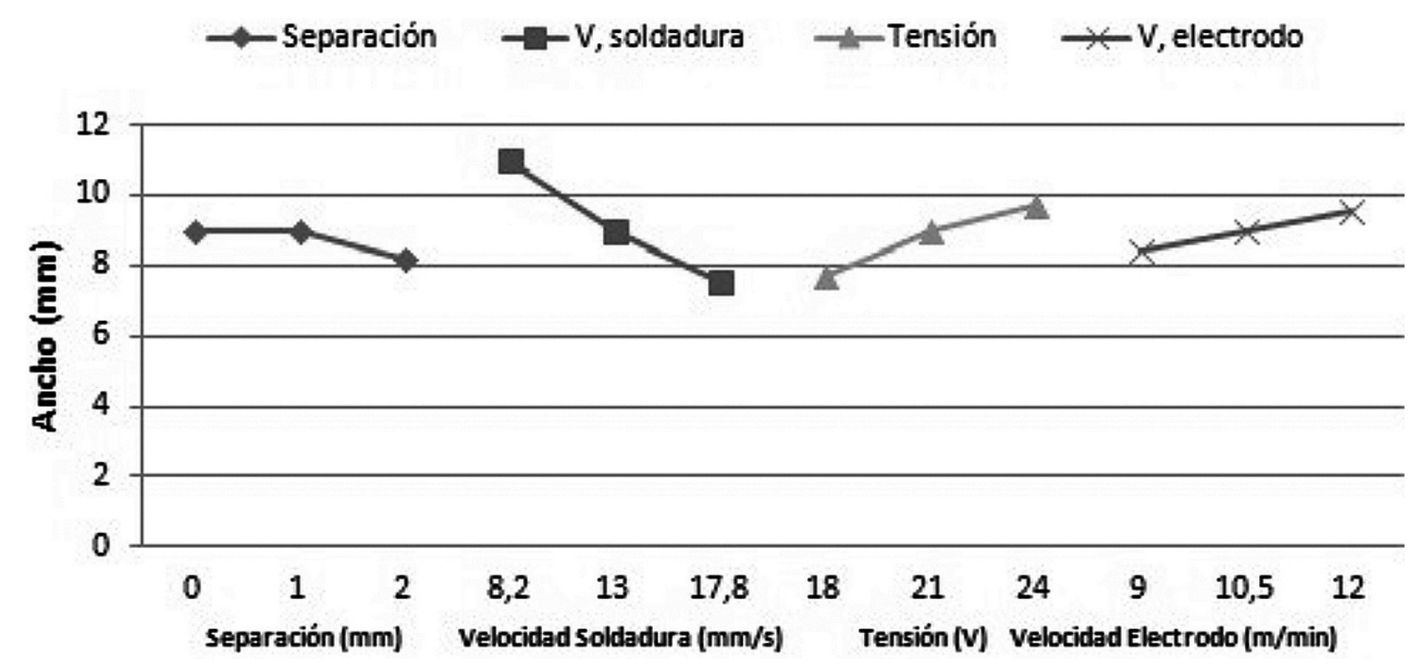

Figura 8. Ancho del cordón en función de los factores tecnológicos del proceso GMAW.

Figure 8. Width of the bead as a function of the technological parameters of the GMAW process. 

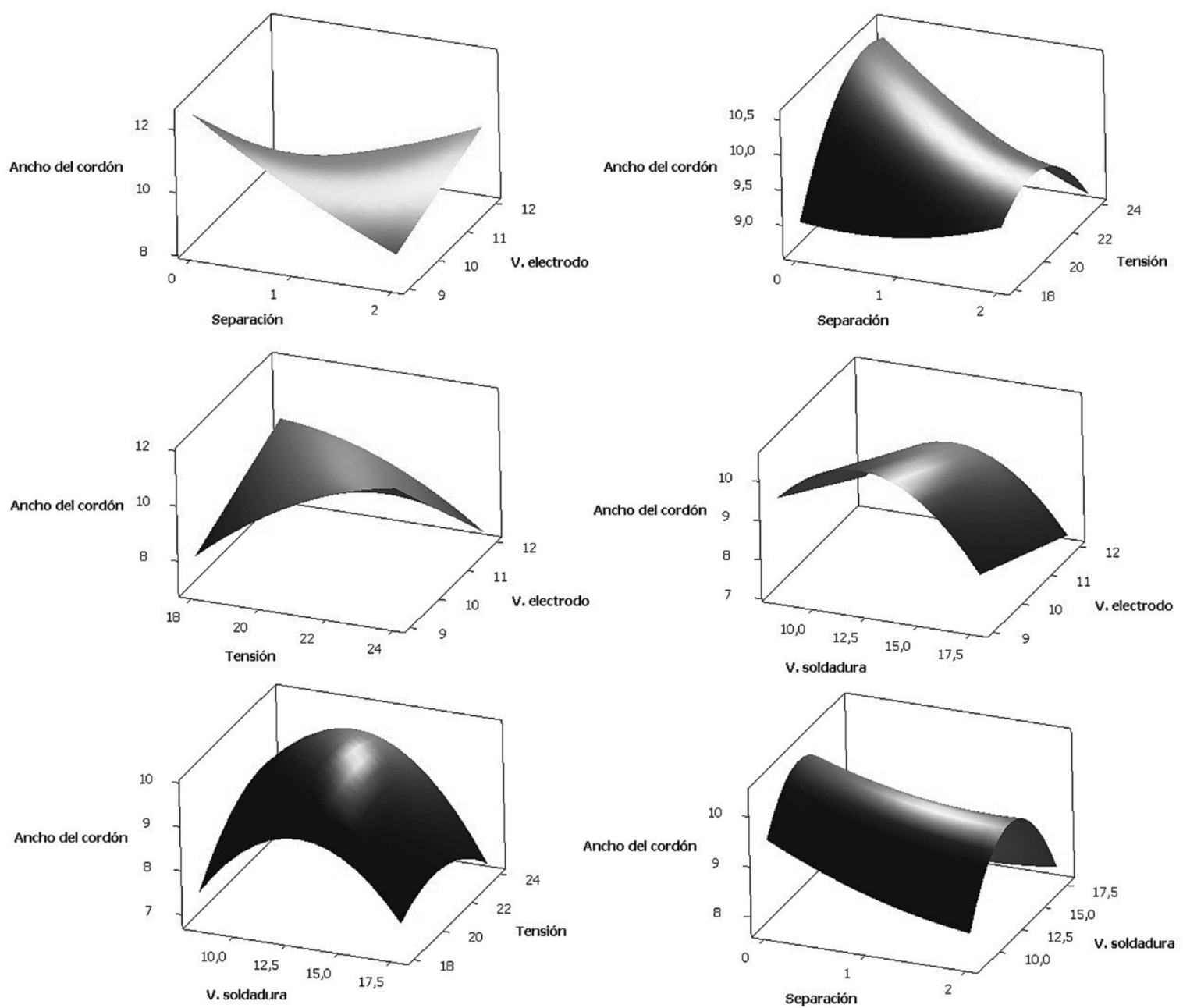

Figura 9. Gráficas de superficie de respuesta del sobreespesor en función de la variación de los factores tecnológicos del proceso GMAW.

Figure 9. Response surface graphs of the overhead as a function of the technological parameters of the GMAW process.

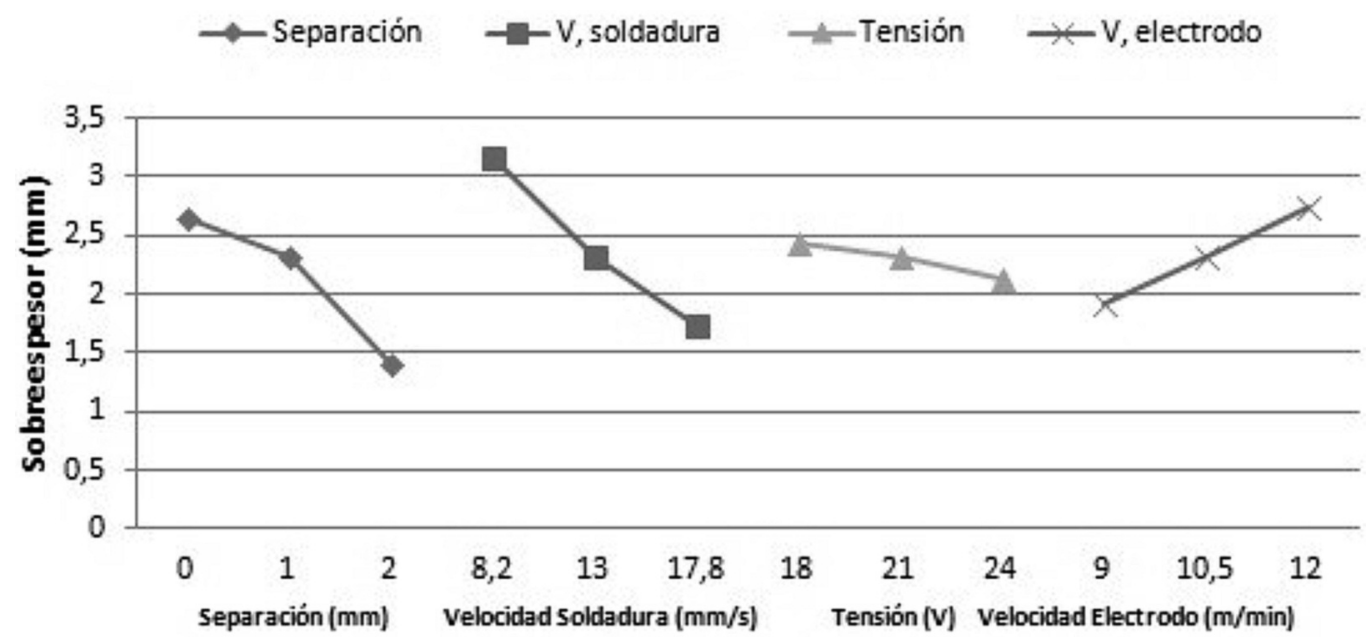

Figura 10. Variación del sobreespesor del cordón de soldadura en función de la variación de los factores tecnológicos del proceso GMAW.

Figure 10. Overhead of the bead as a function of the technological parameters of the GMAW process. 
con las fuerzas de superficie para ángulos de mojado menores. En cualquier caso, la tensión es el factor menos significativo en la variación del sobreespesor.

La ecuación para el ángulo del cordón queda modelizada como se establece en la expresión (8):

$Y_{4}=624,5362+34,6354 S-22,8277 V_{s}-$

$-44,8819 T+8,3542 V_{e}+5,8750 S^{2}+$

$+0,3852 V_{s}^{2}+0,9722 T^{2}-1,5104 S V_{s}-$

$-1,1667 S T+0,5208 V_{s} T-1,1667 S V_{\varepsilon}+$

$+0,1736 V_{s} V_{\varepsilon}-0,3889 T V_{\varepsilon}$

En las figuras 11 y 12 se observa que los factores más determinantes sobre el ángulo del cordón son la velocidad de soldadura, la separación y la tensión, de modo que se obtiene un ángulo mínimo conforme estos factores aumentan.
El ángulo del cordón es consecuencia de la mojabilidad del metal de soldadura sobre el metal base. Así, una buena mojabilidad va asociada a un ángulo de cordón menor. Adiciones de pequeñas cantidades de gases, como hidrógeno por ejemplo, en el gas de protección favorece la mojabilidad y, por tanto, disminuye el ángulo del cordón. Para un gas de protección dado, suele considerarse la tensión como el factor más decisivo para el control de la fluidez del metal y de la mojabilidad de la soldadura sobre el metal base $^{[15]}$. Los resultados de este trabajo indican que la velocidad de soldadura y la separación influyen con un grado de significación similar a la tensión.

Desde el punto de vista de la geometría del cordón, ésta sugiere que existe relación entre el ángulo del cordón y la relación entre el sobreespesor y la anchura del mismo. Así, se ha ensayado una corre-
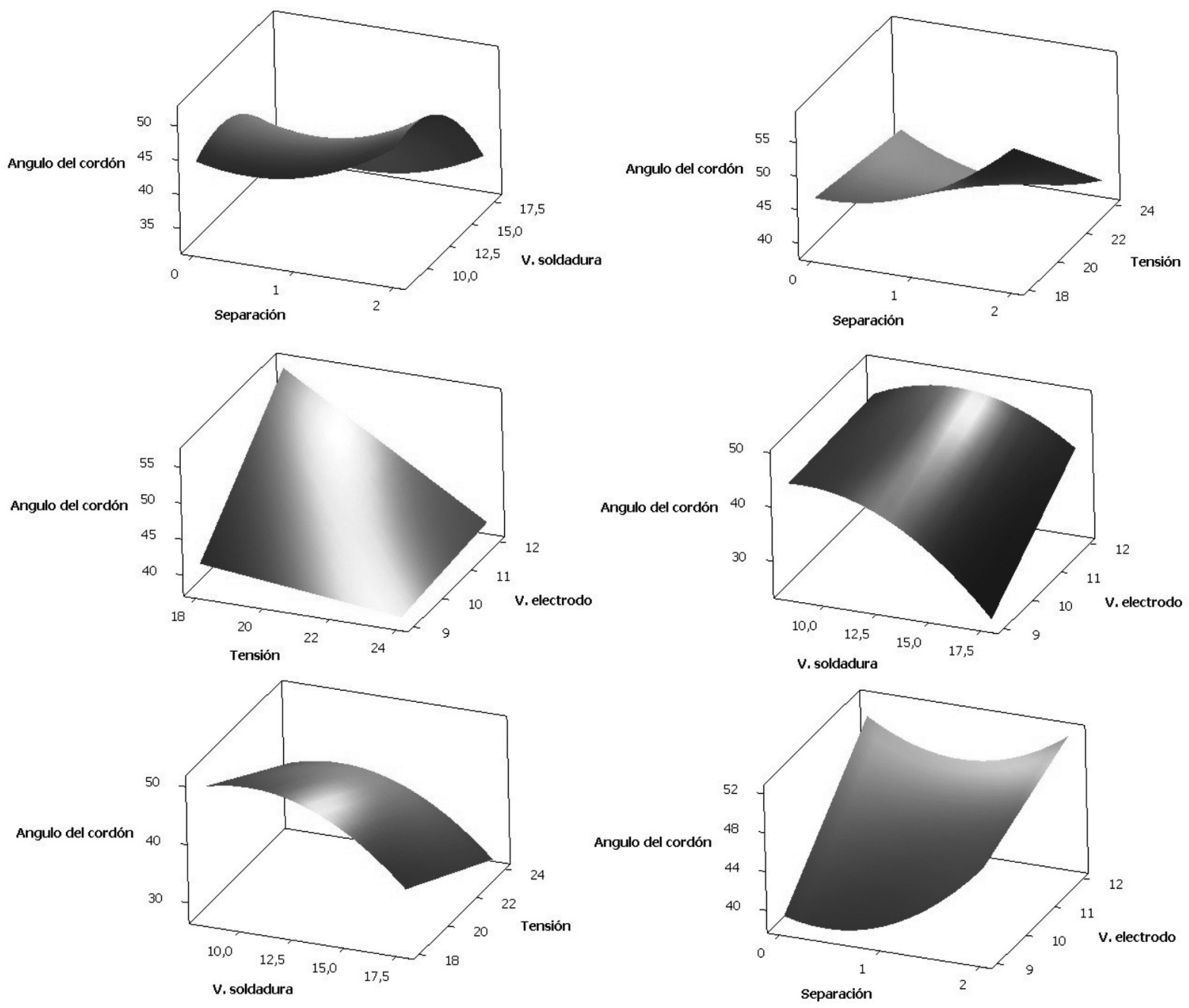

Figura 11. Gráficas de superficie de respuesta del ángulo del cordón en función de la variación de los factores tecnológicos del proceso GMAW.

Figure 11. Response surface graphs of the bead angle as a function of the technological parameters of the GMAW process. 


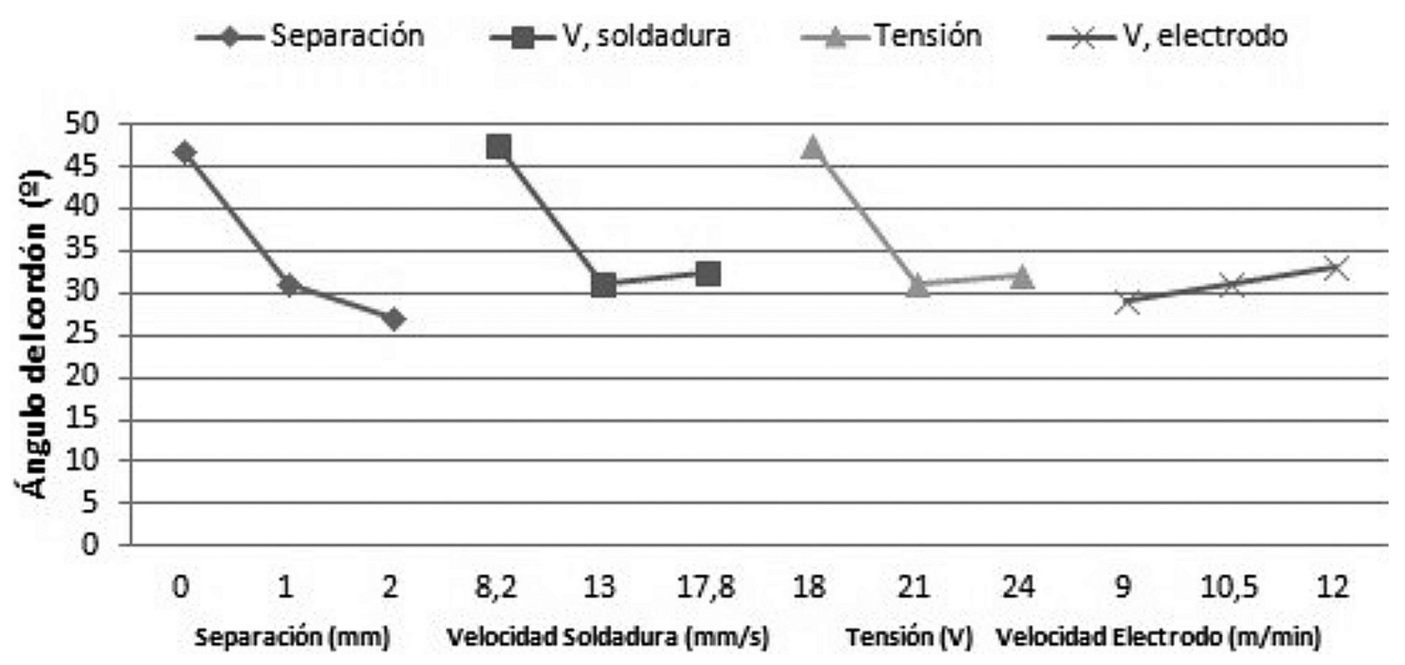

Figura 12. Variación del ángulo del cordón de soldadura en función de la variación de los factores tecnológicos del proceso GMAW.

Figure 12. Bead angle as a function of the technological parameters of the GMAW process.

lación entre el ángulo, $\theta$, y la relación entre el sobreespesor, $\mathrm{S}$ y la mitad de la anchura del cordón, A/2. En la figura 13 se observa la correlación indicada.

Aunque el índice de correlación $\mathrm{R}^{2}$ encontrado no es elevado, resulta muy significativo teniendo en cuenta que se ha realizado el ajuste para todas las variables tecnológicas experimentadas que, como ha quedado demostrado en las figuras 11 y 12 , presentan su propia influencia. Incluso se observa que obviando sólo tres ensayos $(4,10$ y 17$)$ la correlación $\mathrm{R}^{2}$ aumenta hasta 0,86 . Por tanto, se puede considerar, como era esperable, que el ángulo del cordón representa la relación existente entre el sobreespesor y la anchura de dicho cordón.

\section{OPTIMIZACIÓN}

En toda unión soldada interesa maximizar la penetración y minimizar en la medida de lo posible el ancho, el sobreespesor y el ángulo del cordón; se analizan los valores correspondientes a la interacción entre dichos factores. Para esto se utiliza el optimizador de respuesta de Sagata que proporciona una solución óptima para las combinaciones de variables de entrada (separación, tensión, velocidad de soldadura y velocidad de electrodo) y una gráfica de optimización ${ }^{[16]}$. La gráfica de optimización es interactiva; puede ajustar los valores de configuración de variables de entrada en la gráfica a fin de encontrar las soluciones más deseables. La optimización llevada a cabo en el presente trabajo no tiene en cuenta la ponderación de ningún factor, esto es, se ha considerado la misma importancia para todos los factores. Tal y como se muestra en la figura 14, con los valores de tensión de $18 \mathrm{~V}$, una velocidad de soldadura de $17,80 \mathrm{~mm} / \mathrm{s}$, un valor de velocidad de electrodo de $9 \mathrm{~m} / \mathrm{min}$ y una separación entre piezas de $2 \mathrm{~mm}$, se obtienen como valores óptimos de la geometría del cordón los que se reflejan en la tabla VI.

Tabla VI. Valores correspondientes a la geometría del cordón en condiciones de operación óptimas. Contraste de los valores obtenidos por el modelo teórico y los obtenidos experimentalmente

Table VI. Geometry values corresponding to a bead that it has been obtained under optimum operating conditions. Experimental values and theoretical values are compared

\begin{tabular}{lcccc}
\hline Resultados & Penetración $(\mathbf{m m})$ & Ancho $(\mathbf{m m})$ & Sobreespesor $(\mathbf{m m})$ & Ángulo $\left(^{\circ}\right)$ \\
\hline Numérico & 3,64 & 5,66 & 0,26 & 36,17 \\
Experimental & 4,00 & 5,14 & $0-0,20$ & $0-15$ \\
Error & $9 \%$ & $10 \%$ & - & - \\
\hline
\end{tabular}



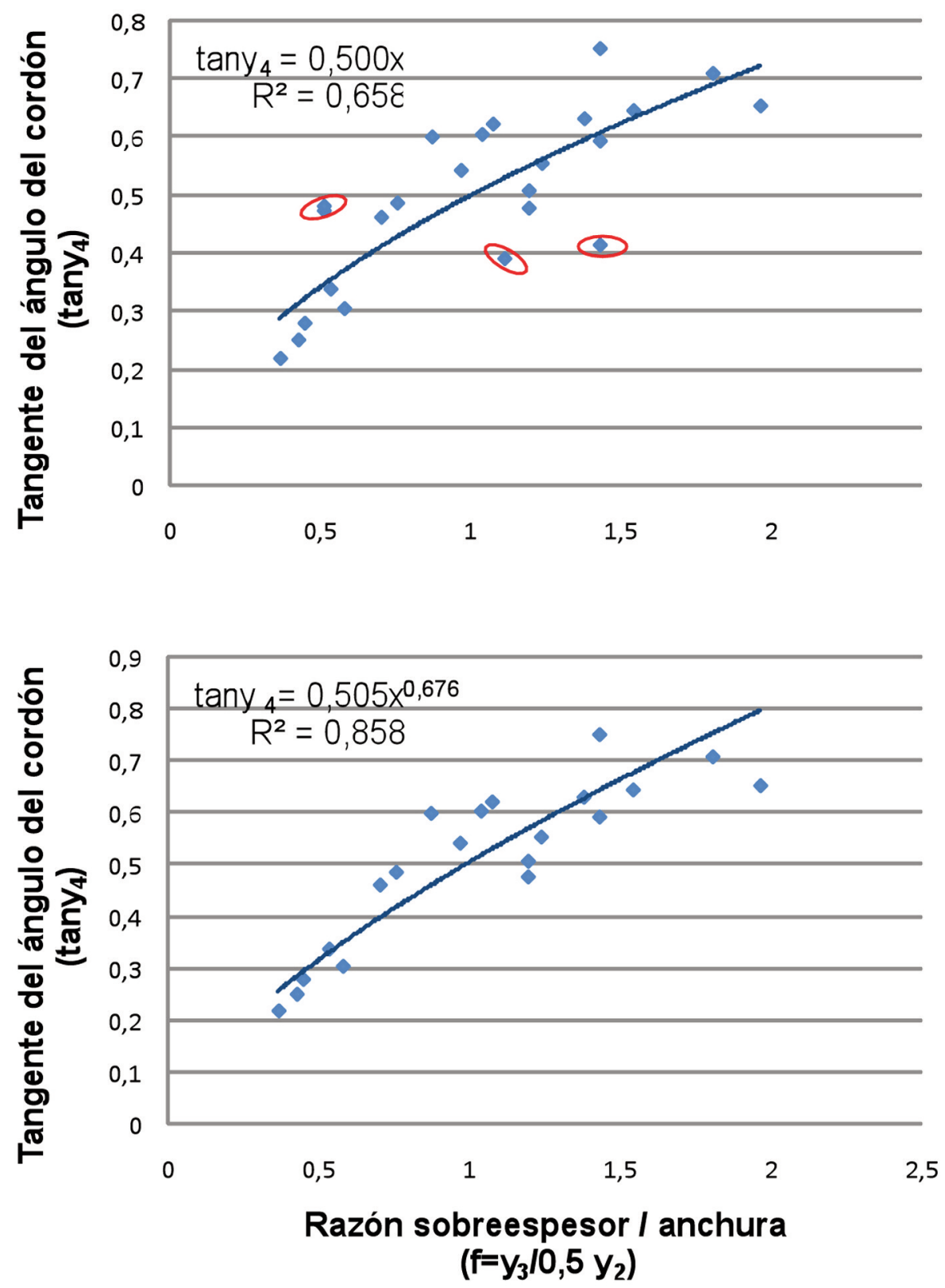

Figura 13. Relación existente entre el ángulo del cordón y la razón sobreespesor/anchura del cordón. La correlación mejora significativamente eliminando sólo tres condiciones de ensayo (puntos señalados con líneas).

Figure 13. Relationship between the bead angle and overhead/ bead widthness ratio. The correlation improves significantly without three results (pointed out with lines).

Al no ponderar ninguna salida no se establece un objetivo de optimización preferente como puede ser buscar una penetración máxima, sino que en la optimización se persigue como objetivo el conjunto de los factores. En estos espesores, no suele tener sentido objetivos de penetración total con soldaduras sin preparación de bordes con una sola pasada por una sola cara, propio de espesores bastante inferiores.
Se ha efectuado un experimento con los parámetros óptimos para la separación, velocidad de soldadura, tensión y velocidad de alimentación del electrodo que se obtienen en la modelización numérica. En la figura 15 se establece un detalle de la geometría del cordón de soldadura obtenido bajo las condiciones de optimización.

En la tabla VI se indican los valores medidos de los diversos elementos de la geometría del cordón de 


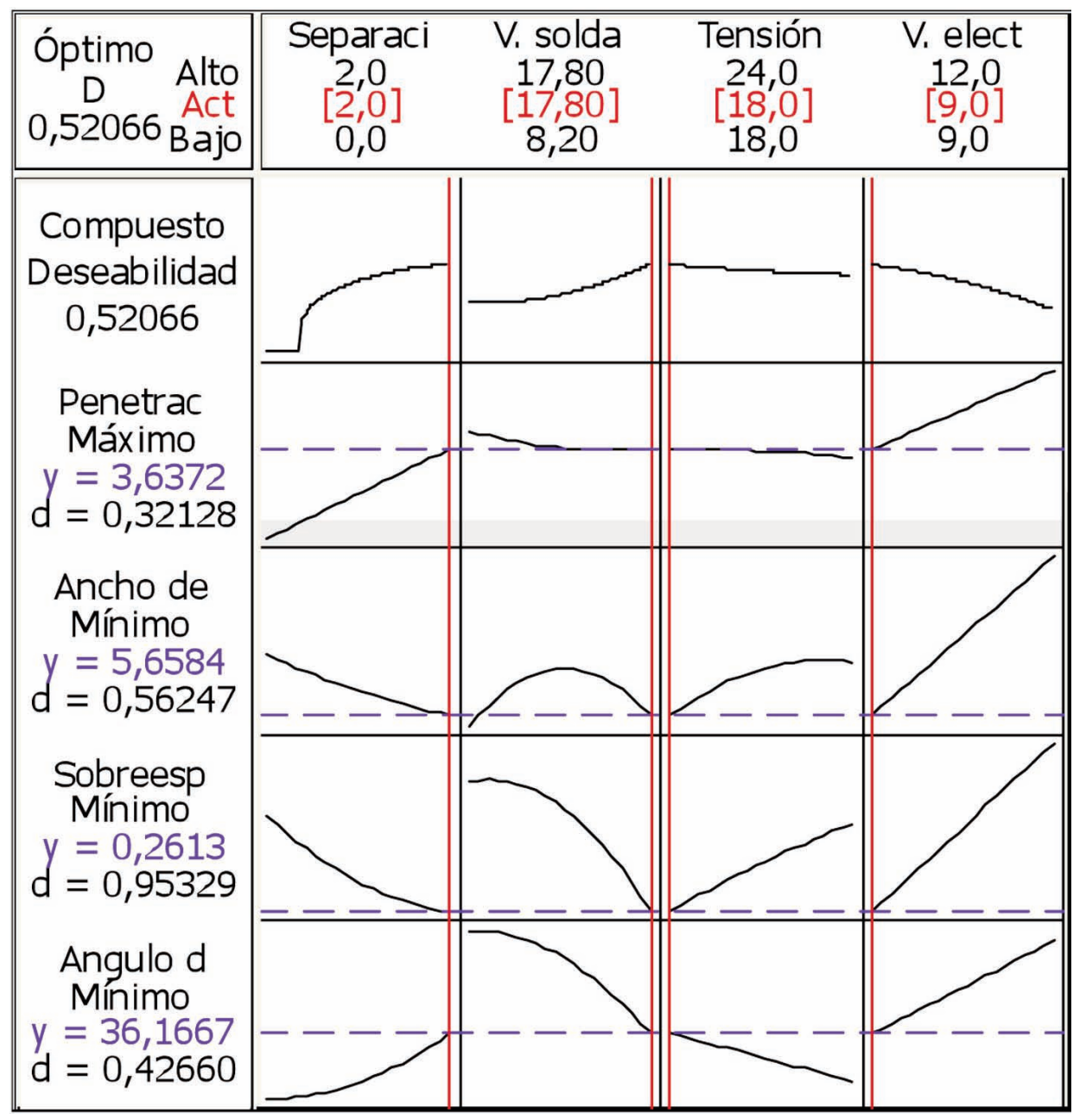

Figura 14. Gráfica correspondiente a los valores óptimos de los parámetros tecnológicos del proceso.

Figure 14. Graphic corresponding to the optimum values for the technological parameters of the GMAW process.

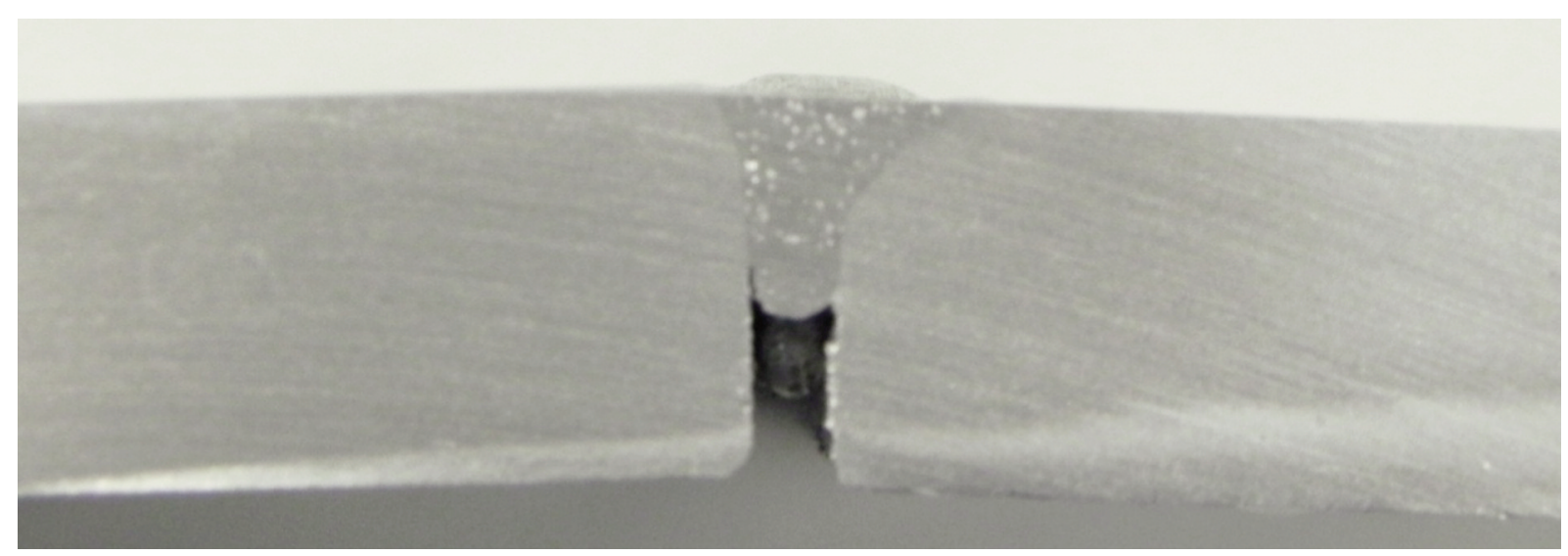

Figura 15. Cordón de soldadura óptimo; tensión $18 \mathrm{~V}$; velocidad de soldadura $17,8 \mathrm{~mm} / \mathrm{s}$; velocidad de electrodo $9 \mathrm{~m} / \mathrm{min}$; separación $2 \mathrm{~mm}$.

Figure 15. Optimum weld; voltage $18 \mathrm{~V}$; welding velocity $17.8 \mathrm{~mm} / \mathrm{min}$; wire feed rate $9 \mathrm{~m} / \mathrm{min}$; butt distance $2 \mathrm{~mm}$. 
soldadura correspondientes a dichas condiciones, así como la comparación entre los valores experimentales y los que arroja el modelo teórico. Como se puede observar, existe una alta correspondencia de los valores establecidos por el modelo con los experimentales. Así, el error medio detectado para la penetración, entre el valor arrojado por el modelo y el experimental para los 24 experimentos realizados fue $12,75 \%$; el error observado en el ensayo de optimización es inferior a dicho valor. También se observa una buena aproximación para la anchura del cordón. En relación al sobreespesor y al ángulo de mojado, puede afirmarse que cuanto menores sean los valores obtenidos, mejor será el comportamiento de la unión, cuestión que es reafirmada por los resultados experimentales.

\section{CONCLUSIONES}

- En la soldadura por arco eléctrico, interesa maximizar la penetración y minimizar el ancho, el sobreespesor y el ángulo del cordón. Se realiza un diseño experimental con la metodología de superficie de respuesta. Con la aplicación MSR se obtienen las ecuaciones de la modelización matemática de la penetración, sobreespesor, ancho de cordón y ángulo de cordón. Para la penetración los factores más determinantes son la separación y la velocidad de alimentación del electrodo; se obtiene una mayor penetración conforme aumentan la separación y la velocidad de alimentación del electrodo. En el análisis del ancho del cordón, el factor más determinante es la velocidad de soldadura, de modo que se obtiene un ancho de cordón menor conforme dicha velocidad sea mayor. En el caso del sobreespesor, la separación entre las piezas y la velocidad de soldadura son los dos factores más importantes. El valor mínimo para el sobreespesor corresponde a los mayores valores de la separación y de la velocidad de soldadura. En el análisis del ángulo del cordón, los factores a controlar son la tensión y la separación entre las piezas ya que valores elevados de éstos conducen a ángulos mínimos de mojado. En relación a este último parámetro, se ha demostrado que presenta una buena correlación con la relación sobreespesor/anchura del cordón a pesar de la naturaleza del proceso, que difiere de los procesos típicos de mojado por gravedad.

- Se han hallado los valores para la separación, tensión, velocidad de alimentación del electrodo y velocidad de soldeo que optimizan las funciones de respuesta, esto es, que conducen a una profundidad máxima junto con valores mínimos para el sobreespesor, anchura y ángulo del cordón. Dichos valores se indican en la tabla VI.

- Experimentalmente se valida el procedimiento propuesto mediante la medición de la geometría del cordón para las condiciones de soldeo obtenidas teóricamente. La comparación entre los valores teóricos y experimentales indica un grado de concordancia adecuado entre ambos. Se observa que los errores obtenidos no sobrepasan el $10 \%$, valores inferiores a los obtenidos por otros autores mediante diversas funciones de correlación. Por tanto, se puede afirmar que la modelización numérica diseñada y desarrollada predice adecuadamente la geometría del cordón de soldadura.

\section{Agradecimientos}

Los autores agradecen la ayuda en el desarrollo del procedimiento experimental al Instituto Politécnico de Cartagena, al Dpto. de Materiales e Ingeniería Metalúrgica del IDR de la Universidad de CastillaLa Mancha.

\section{REFERENCIAS}

[1] B.K. Srivastava, S.P. Tewar y J. Prakash, Int. J. Engineer. Sci. Technol. 2 (2010) 1.425-1.432.

[2] AWS, Welding Handbook, Vol. 2. Welding Processes, 8th ed., 2001, pp. 47-65.

[3] A.M. Al-Mukhtar, H. Biermann, P. Hübner y S. Henkel, J. Mater. Eng. Perform. 19 (2010) 1.225-1.234.

[4] G. Kumar y K.N. Prabhu, Adv. Colloid. Interfac. 133 (2007) 61-89.

[5] J.C. Mc Glone y D.B. Chadwick, Welding Institute Report 80 (1978) 256-263.

[6] E. Karadeniz, U. Ozsarac y C. Yildiz, Mater. Design. 28 (2007) 649-656.

[7] M.A. Wahab y M.J. Painter, J. Pm. Ves. \& Piping 73 (1997) 153-159.

[8] I.S. Kim, C.E. Park, Y.J. Jeong y J.S. Son, Int. J. Adv. Manuf. Techn. 18 (2001) 98-102.

[9] I.S. Kim, J.S. Son, I.G. Kim, J.Y. Kim y O.S. Kim, J. Mater. Process. Tech. 136 (2003) 139-145.

[10] P.E. Murray y A. Scotti, Sci. Technol. Weld. Joi. 4 (1999) 112-117.

[11] M. Dorta, J. Vidal, A. Mateo, G. Fargas y F. Camejo, Dyna 78 (2011) 206-215.

[12] M.C. Payares, C. De Barros, P. Muñoz y Z. Cassier, Rev. Latinoamericana de Metalurgia y Materiales 17 (1997) 5-12. 
[13] V. Aleo, M.K.S. Madugula y R. Balachandar, Canadian Journal of Civil Engineering Publisher 33 (2006) 151-160.

[14] V. Miguel, A. Martínez, M.C Manjabacas, J. Coello y A. Calatayud, AIP Conference Proceedings, 1, New York, USA, AMER INST. PHYSICS, 2009, pp. 170-179.
[15] D. Hoffman, K. Dable y D. Fisher, Welcome to welding, Ed. Pearson Education Limited ), USA, 2010, pp. 69-75.

[16] M. Estrems, E.J. Martínez-Conesa y V. Miguel, Dyna 84 (2009) 251-258. 